\title{
Pulmonary fungus ball caused by Penicillium capsulatum in a patient with type 2 diabetes: a case report
}

\author{
Min Chen ${ }^{1 \dagger}$, Jos Houbraken ${ }^{2 \dagger}$, Weihua Pan ${ }^{1 \dagger}$, Chao Zhang ${ }^{1}$, Hao Peng ${ }^{3}$, Lihui $\mathrm{Wu}^{3}$, Deqiang Xu ${ }^{4}$, Yiping Xiao ${ }^{4}$, \\ Zhilong Wang ${ }^{3}$ and Wanqing Liao ${ }^{1^{*}}$
}

\begin{abstract}
Background: Following the recent transfer of all accepted species of Penicillium subgenus Biverticillium to Talaromyces (including Talaromyces marneffei, formerly Penicillium marneffei), Penicillium species are becoming increasingly rare causal agents of invasive infections. Herein, we present a report of a type 2 diabetes patient with a fungus ball in the respiratory tract caused by Penicillium capsulatum.

Case presentation: A 56-year-old Chinese female gardener with a 5-year history of type 2 diabetes presented at the Shanghai Changzheng Hospital with fever, a cough producing yellow-white sputum, and fatigue. The therapeutic effect of cefoxitin was poor. An HIV test was negative, but the $\beta$-D-glucan test was positive (459.3 pg/ $\mathrm{ml}$ ). Chest radiography revealed a cavitary lesion in the left upper lobe, and a CT scan showed globate cavities with a radiopaque, gravity-dependent ball. The histopathologic features of the tissue after haematoxylin-eosin staining showed septate hyphae. The fungus was isolated from the gravity-dependent ball and identified as Penicillium capsulatum based on the morphological analysis of microscopic and macroscopic features and on ribosomal internal transcribed spacer sequencing. After surgery, the patient was cured with a sequential treatment of fluconazole $400 \mathrm{mg}$ per day for 90 days and caspofungin $70 \mathrm{mg}$ per day for 14 days.

Conclusions: Although the prognosis is often satisfactory, clinicians, mycologists and epidemiologists should be aware of the possibility of infection by this uncommon fungal pathogen in diabetes patients, since it may cause severe invasive infections in immunocompromised hosts such as diabetes and AIDS patients.
\end{abstract}

Keywords: Fungal ball, Pulmonary infections, Penicillium capsulatum

\section{Background}

Penicilliosis is an invasive fungal infection that primarily occurs in the southeast and eastern regions of Asia, including Thailand, northeast India, China, Hong Kong, Laos, Cambodia, Malaysia, Myanmar, Vietnam and Taiwan [1]. Penicillium marneffei causes the vast majority of penicillioses, especially in immunosuppressed hosts such as AIDS patients and otherwise healthy individuals [1-3]. This species was recently transferred to the genus Talaromyces together with other Penicillium species belonging to the subgenus Biverticillium [4].

\footnotetext{
* Correspondence: liaowanqing@sohu.com

${ }^{\dagger}$ Equal contributors

'Shanghai Key Laboratory of Molecular Medical Mycology, Department of Dermatology, Shanghai Changzheng Hospital, Shanghai, China

Full list of author information is available at the end of the article
}

Thus, excluding Talaromyces marneffei (formerly known as $P$. marneffei), systemic infections caused by Penicillium species are becoming increasingly rare. Currently, approximately 300 species are grouped within the genus Penicillium, and only a limited number are associated with invasive fungal infections [5]. The majority of Penicillium species are saprobic and commonly occur in soil; however, some species are known for their positive or negative effects on humans. The positive impacts include their use in food fermentation and the production of drugs, and the negative effects are related to the production of mycotoxins, the induction of hypersensitivity reactions (e.g., asthma and extrinsic allergic alveolitis) and the infection of humans. Presently, China has an increasing burden of diabetes (92.4 million adults above the age of 20) [6], and

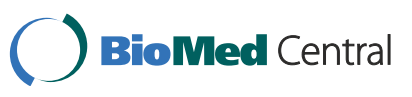




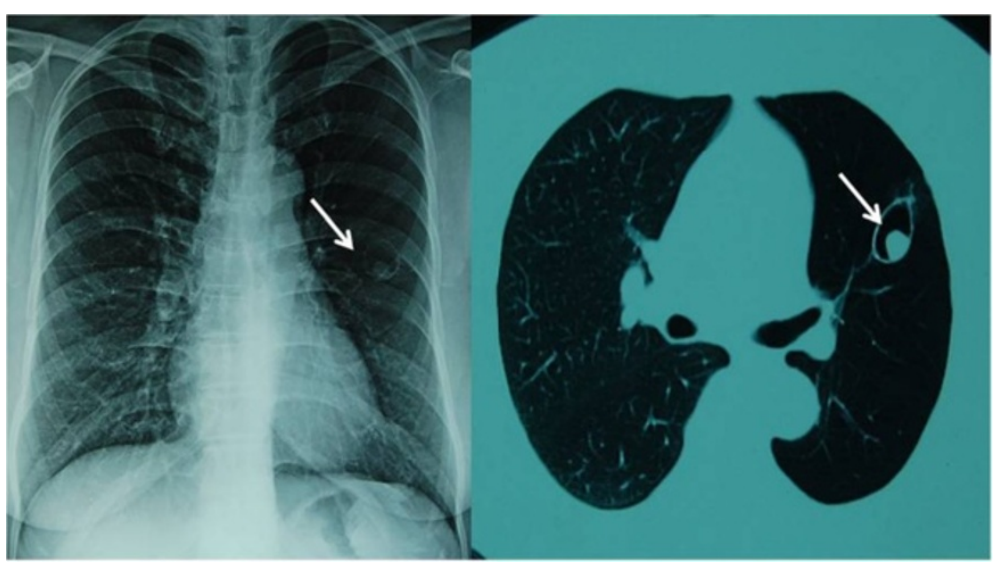

Figure 1 Chest computed tomography showing a fungus ball in the left upper lobe of the lung.

diabetes mellitus is considered to be a significant suppressor of the human immune system. Herein, we report a case of a 56-year-old patient with type 2 diabetes who developed a fungus ball in the left lung caused by Penicillium capsulatum. The diagnosis, treatment and clinical importance of $P$. capsulatum are discussed.

\section{Case presentation}

A 56-year-old Chinese female gardener presented to the Shanghai Changzheng Hospital with fever, a cough producing yellow-white sputum and fatigue. The therapeutic effect of cefoxitin ( $4000 \mathrm{mg} /$ day, 14 days) was poor. She had a 5-year history of type 2 diabetes with effective treatment by insulin injection but no history of tuberculosis, chronic obstructive pulmonary disease, asthma or other underlying immunosuppressive diseases. A test for the human immunodeficiency virus was negative, but the $\beta$-D-glucan test was positive $(459.3 \mathrm{pg} / \mathrm{ml})$. Chest radiography revealed a cavitary lesion in the left upper lobe, and chest computed tomography (CT) showed globate cavities with a radiopaque, gravity-dependent ball (Figure 1). Based on these data, the causal agent of the infection was initially thought to be Aspergillus spp. because of the fungus ball's resemblance to an aspergilloma caused by species such as Aspergillus fumigatus. Histologic sections of the tissue were stained with haematoxylin and eosin (HE) and showed septate fungal hyphae (Figure 2). Specimens from the gravity-dependent ball were obtained, and the fungal strain was identified as Penicillium capsulatum via microand macro-morphology examination and molecular sequence analysis. For macroscopic examination, the culture was grown for 7 days at $25^{\circ} \mathrm{C}$ on malt extract agar (MEA), Czapek yeast extract agar (CYA) and yeast extract sucrose agar (YES), and the fungus exhibited restricted growth on all media $(13-17 \mathrm{~mm})$ with grey-green sporulating colonies. Growth on MEA plates incubated at $30^{\circ} \mathrm{C}$ and $37^{\circ} \mathrm{C}$ was faster $(17-25 \mathrm{~mm})$ than at $25^{\circ} \mathrm{C}$. Microscopy analysis of the colonies grown on MEA showed short, smoothwalled, monoverticillate Penicillium conidiophores. The conidia were smooth walled and ellipsoid to slightly cylindrical and measured 3.5-4.5 × 2.5-3.0 $\mu \mathrm{m}$ (Figure 3). Molecular characterisation of the strain was performed by PCR amplification and sequencing of the ribosomal internal transcribed spacer region as described by Houbraken et al. [7]. The isolate was deposited in the CBS collection under the accession number CBS 134186, and newly generated sequences were deposited in GenBank. Figure 4 shows that the strain belongs to Penicillium section Ramigena [5] and that it is closely related to the type culture of Penicillium capsulatum, CBS 301.48. So we diagnosed this infection as Penicillioma which we created particularly. After pulmonary lobectomy, the patient was cured sequentially by

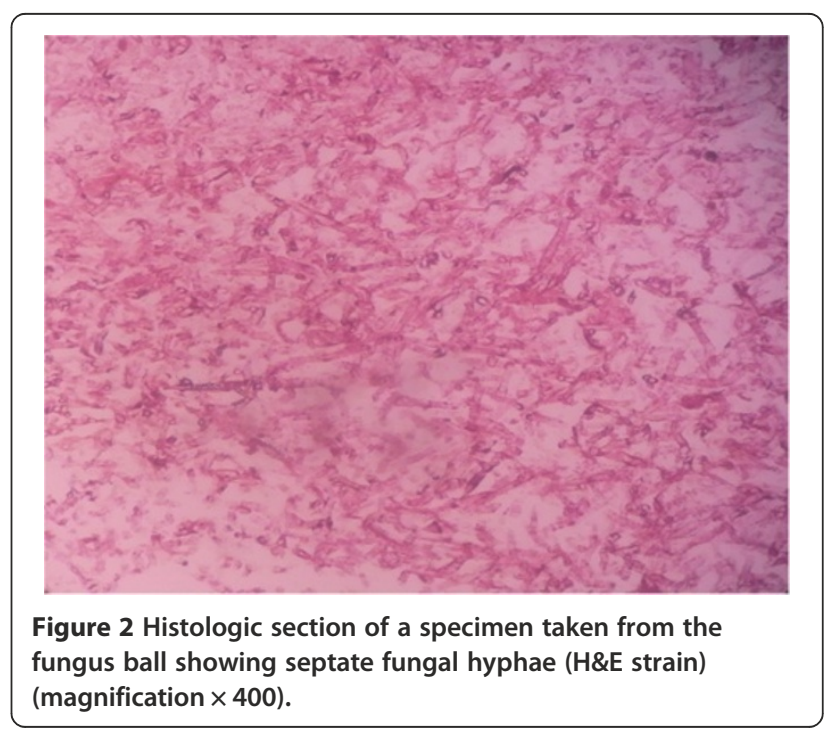



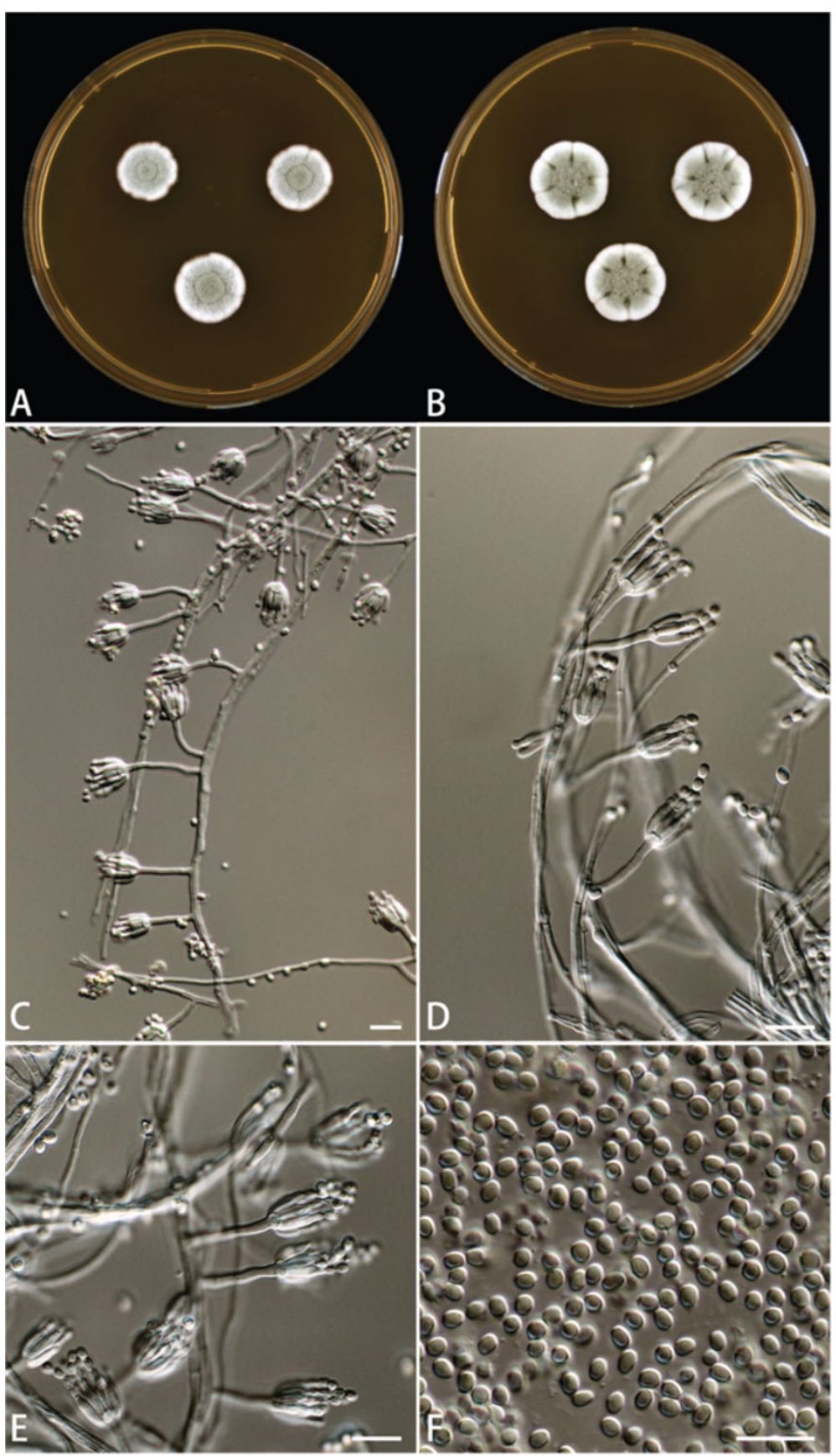

Figure 3 Penicillium capsulatum CBS 134186. A. 7 days old culture on MEA incubated at $25^{\circ} \mathrm{C}$. B. 7 days old culture on MEA incubated at $37^{\circ} \mathrm{C}$. C-E. Conidiophores. F. Conidia. Scale bars $=10 \mu \mathrm{m}$.

treatment of fluconazole (400 mg/day, 90 days) and caspofungin (70 mg/day, 14 days).

Furthermore, to confirm the antifugal susceptibility in vitro, the clinical isolate CBS 134186 was, together with other environmental $P$. capsulatum isolates, tested in vitro against seven antifungal agents (amphotericin $\mathrm{B}$, voriconazole, itraconazole, ketoconazole, fluconazole, 5-fluorocytosine, and caspofungin) using the CLSI M38-A2 broth microdilution method [8]. The results of this analysis are summarized in Table 1 and show that all tested $P$. capsulatum strains have a low minimal effective concentration (MEC) to caspofungin and a high minimal inhibitory concentration (MIC) to fluconazole. No substantial difference for all seven antifungal agents 


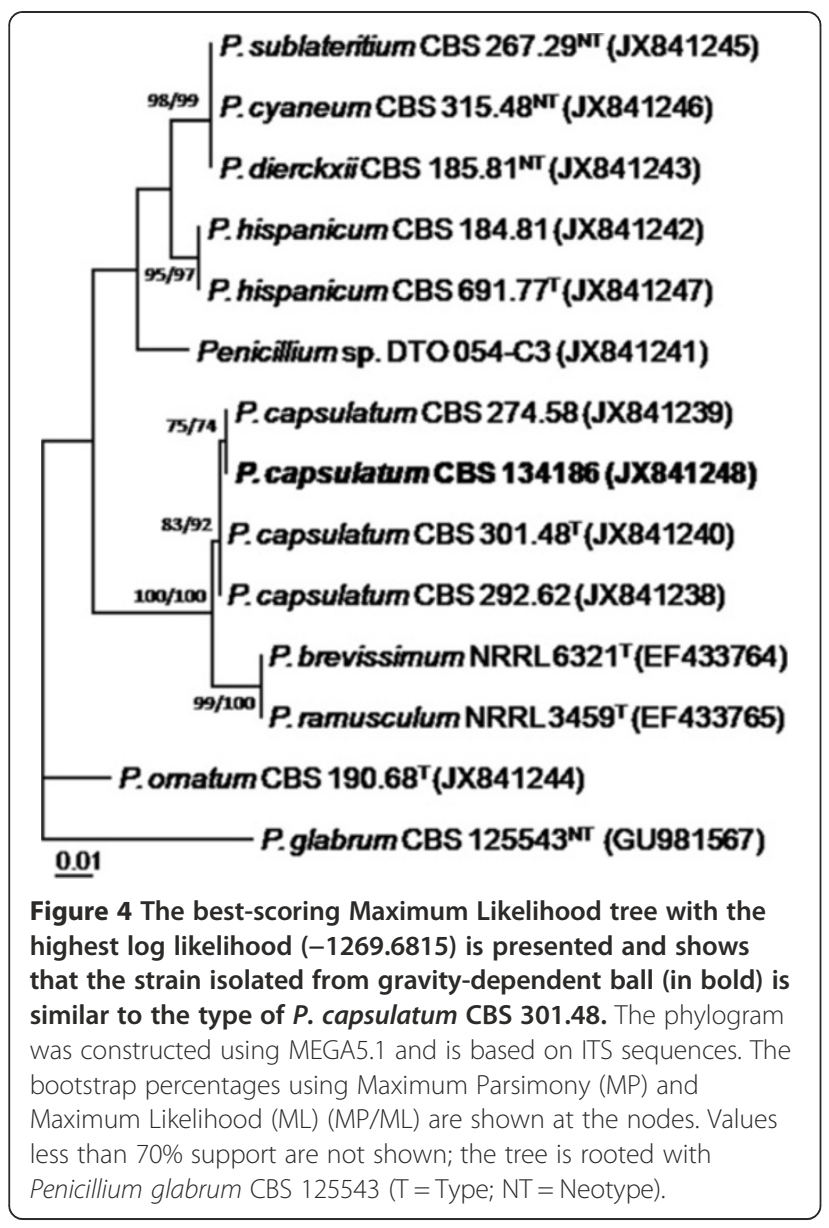

in antifungal susceptibility was observed between CBS 134186 and the $P$. capsulatum strains isolated from environmental sources (Table 1).

\section{Conclusions}

As reviewed by Lyratzopoulos et al. [9], only fifteen cases of invasive infections caused by species other than T. marneffei have been linked to the Penicillium genus worldwide. More recently, other Penicillium species, such as $P$. chrysogenum, $P$. piceum and $P$. purpurogenum, were found to be associated with these infections [10-13]. However, when applying the current taxonomic concepts [4], the latter two Penicillium species now belong to the Talaromyces genus, namely $T$. purpurogenus and T. piceus.

Penicillium capsulatum, a rare species belonging to Penicillium section Ramigena [5], is considered a useful microorganism in the paper manufacturing industry $[14,15]$, but it has never been previously recognised as a causative agent of invasive infection in humans. One of the pathogenicity factors of Penicillium species causing invasive infections is its ability to grow at $37^{\circ} \mathrm{C}$ [16]. The majority of Penicillium species have a maximum growth temperature below $37^{\circ} \mathrm{C}$; however, exceptions include $P$. chrysogenum, $P$. citrinum, $P$. decumbens and $P$. janthinellum, and some of these species are causal agents of invasive infections, as stated above $[10,16]$. Our clinical P. capsulatum isolate grows well on MEA, CYA and YES incubated at $37^{\circ} \mathrm{C}$, and growth at $30^{\circ} \mathrm{C}$ and $37^{\circ} \mathrm{C}$ was faster (17$25 \mathrm{~mm})$ than at $25^{\circ} \mathrm{C}(13-17 \mathrm{~mm})$. In our opinion, both the fungus's ability to grow at $37^{\circ} \mathrm{C}$ and the patient's diabetes mellitus contributed to the presence of the fungus ball in the patient. Despite the clinical strain can be considered resistant to fluconazole, the treatment seemed to be effective in vivo in this case. We believe that combined antifungal drug (caspofungin + fluconazole), resection of the primary lesion, and the patient's immune status are important for satisfactory prognosis. Additionally, there is no substantial difference in antifungal susceptibilities between the $P$. capsulatum strains isolated from environmental sources and the CBS 134186 isolate (Additional file 1: Table S1).

In the present study, we used phenotypic characters and ITS sequences for identification. Identification based solely on the phenotype is often difficult and requires well-trained staff. Currently, molecular-based techniques, especially DNA sequencing, are frequently used for identification, and the ITS region is accepted as the primary fungal barcode [17]. This study shows that $P$. capsulatum can be identified based on ITS sequence data alone. In contrast, other Penicillium (and Aspergillus) species cannot be unambiguously identified by ITS sequencing alone, and partial $\beta$-tubulin or calmodulin sequences are required to ensure correct species identification [7]. The initial results based on the chest radiography and CT scan showed structures resembling an aspergilloma caused by Aspergillus. Penicillium and Aspergillus are sister genera and belong to the family Aspergillaceae [12]. This close relationship may explain their resemblance in chest radiographs and CT scans. It should be noted that Aspergillus species cause most pulmonary mycetomas, and other phylogenetically unrelated fungi including Fusarium, Mucor

Table 1 Antifungal susceptibilities of $P$. capsulatum isolates from strains isolated from environmental sources and CBS $134186(\mu \mathrm{g} / \mathrm{mL})$

\begin{tabular}{|c|c|c|c|c|c|c|c|}
\hline & Amphotericin B & Voriconazole & Itraconazole & Ketoconazole & Fluconazole & 5-fluorocytosine & Caspofungin \\
\hline ATCC10420 & 1 & 1 & 0.25 & 0.125 & $>64$ & 16 & 1 \\
\hline ATCC48735 & 1 & 0.5 & 0.25 & 0.0625 & $>64$ & 32 & 1 \\
\hline CBS134186 & 0.5 & 0.5 & 0.25 & 0.0625 & $>64$ & 32 & 1 \\
\hline
\end{tabular}


and Paecilomyces, can cause similar clinical manifestations [18].

In summary, Penicillium capsulatum produced a pulmonary fungus ball in a female patient with type 2 diabetes but was cured with combined surgical and antifungal treatment. An aggressive diagnostic and therapeutic strategy should be pursued for such infections. To our knowledge, this is the first report of a pulmonary infection caused by $P$. capsulatum worldwide; thus, this report expands our understanding of the pathogenicity of the genus Penicillium in this age of global warming [19]. Clinicians, mycologists and epidemiologists should be aware of the possibility of infection by uncommon fungal pathogens in patients because emerging pathogenic fungi are increasingly recognised as major threats to human health $[19,20]$.

\section{Consent}

Written informed consent was obtained from the patient for publication of this Case report and any accompanying images. A copy of the written consent is available for review by the Series Editor of this journal.

\section{Additional file}

Additional file 1: Table S1. Antifungal susceptibilities of $P$. capsulatum isolates from environmental or clinical sources $(\mu \mathrm{g} / \mathrm{mL})$.

\section{Competing interests}

The authors declare that they have no competing interests.

\section{Authors' contributions}

MC collected the clinical data, performed part of molecular identification and drafted the manuscript. JH performed the phenotypic and molecular identification and helped with the draft of the manuscript. WP carried out the histologic examinations. CZ performed the susceptibility test. HP collected the clinical specimen and the clinical data. LW and ZW collected the clinical specimen. DX and YX performed the phenotypic identification. WL supervised the clinical case interpretation, participated in the coordination and concept of the manuscript, and helped with the draft of the manuscript. All authors have read and approved the manuscript.

\section{Acknowledgments}

We thank Dr. Shuwen Deng and Hongling Jia (Shanghai Changzheng Hospital, Second Military Medical University, China) for her contribution in the isolation of the fungal strain and the reviewers are thanked for the valuable suggestions. This study was funded in part with the grants from the 973 Program (2013CB531601), Shanghai Science and Technology counsel projects of 10DZ22201000, and National Natural Science Foundation of China (No. 31170139 and 81201269).

\footnotetext{
Author details

${ }^{1}$ Shanghai Key Laboratory of Molecular Medical Mycology, Department of Dermatology, Shanghai Changzheng Hospital, Shanghai, China. ${ }^{2}$ Department of Applied and Industrial Mycology, CBS-KNAW Fungal Biodiversity Centre, Utrecht, the Netherlands. ${ }^{3}$ Department of Thoracic Surgery, Shanghai Changzheng Hospital, Shanghai, China. ${ }^{4}$ The College of Life Science, Fudan University, Shanghai, China.
}

\section{References}

1. Chakrabarti A, Slavin MA: Endemic fungal infections in the Asia-Pacific region. Med Mycol 2011, 49(4):337-344.

2. Lee PP, Chan KW, Lee TL, Ho MH, Chen XY, Li CH, Chu KM, Zeng HS, Lau YL: Penicilliosis in children without HIV infection-are they immunodeficient? Clin Infect Dis 2012, 54(2):e8-e19.

3. Hu Y, Zhang J, Li X, Yang Y, Zhang Y, Ma J, Xi L: Penicillium marneffei infection: an emerging disease in mainland China. Mycopathologia 2013, 175(1-2):57-67.

4. Samson RA, Yilmaz N, Houbraken J, Spierenburg H, Seifert KA, Peterson SW, Varga J, Frisvad JC: Phylogeny and nomenclature of the genus talaromyces and taxa accommodated in penicillium subgenus biverticillium. Stud Mycol 2011, 70(1):159-183.

5. Houbraken J, Samson RA: Phylogeny of penicillium and the segregation of trichocomaceae into three families. Stud Mycol 2011, 70(1):1-51.

6. Yang SH, Dou KF, Song WJ: Prevalence of diabetes among men and women in China. N Engl J Med 2010, 362(25):2425-2426. author reply 2426.

7. Houbraken J, Frisvad JC, Samson RA: Taxonomy of penicillium section citrina. Stud Mycol 2011, 70(1):53-138.

8. Wayne D: Reference method for broth dilution antifungal susceptibility testing of yeasts; approved standard-third edition. Clinical and Laboratory Standards Institute 2008, 28(16):1-52.

9. Lyratzopoulos G, Ellis M, Nerringer R, Denning DW: Invasive infection due to penicillium species other than P. marneffei. J Infect 2002, 45(3):184-195.

10. Geltner C, Lass-Florl C, Bonatti H, Muller L, Stelzmuller I: Invasive pulmonary mycosis due to penicillium chrysogenum: a new invasive pathogen. Transplantation 2013, 95(4):e21-e23.

11. Barcus AL, Burdette SD, Herchline TE: Intestinal invasion and disseminated disease associated with penicillium chrysogenum. Ann Clin Microbiol Antimicrob 2005, 4:21.

12. Santos PE, Piontelli E, Shea YR, Galluzzo ML, Holland SM, Zelazko ME, Rosenzweig SD: Penicillium piceum infection: diagnosis and successful treatment in chronic granulomatous disease. Med Mycol 2006, 44(8):749-753.

13. Weng CH, Wang RC, Hsieh TY, Tsai CA, Lin TH: Penicillium pneumonia in a patient with newly diagnosed Franklin disease. Am J Med Sci 2012, 344(1):69-71.

14. Paice MGJL: Removing hemicelluloses from pulps by specific enzymic hydrolysis. J Wood Chem Technol 1984, 4:187-198.

15. Atienza J, Hernandez E, Primo J: Isolation and identification of ethisolide as an antibiotic product from penicillium capsulatum. App/ Microbiol Biotechnol 1992, 37(3):298-300.

16. Pitt J: The current role of aspergillus and penicillium in human and animal health. J Med Vet Mycol 1994, 32(Suppl 1):17-32.

17. Schoch CL, Seifert KA, Huhndorf S, Robert V, Spouge JL, Levesque CA, Chen W: Nuclear ribosomal internal transcribed spacer (ITS) region as a universal DNA barcode marker for fungi. Proc Natl Acad Sci U S A 2012, 109(16):6241-6246.

18. Sigler LKM: Aspergillus, Fusarium, and other opportunistic moniliaecious fungi. 7th edition. Washington DC: American Society for Microbiology Press; 1999

19. Garcia-Solache MA, Casadevall A: Global warming will bring new fungal diseases for mammals. MBio 2010, 1(1):e00061-10.

20. Fisher MCHD, Briggs CJ, Brownstein JS, Madoff LC, McCraw SL, Gurr SJ: Emerging fungal threats to animal, plant and ecosystem health. Nature 2012, 484:186-194.

doi:10.1186/1471-2334-13-496

Cite this article as: Chen et al:: Pulmonary fungus ball caused by

Penicillium capsulatum in a patient with type 2 diabetes: a case report. BMC Infectious Diseases 2013 13:496. 